\title{
Increased yield of finger jointed structural timber by accounting for grain orientation utilizing the tracheid effect
}

\author{
${\text { Anders } \text { Olsson }^{1}\left[\text {. } \cdot \text { Andreas Briggert }^{1} \cdot J^{\prime} \text { an Oscarsson }\right.}^{1}$
}

Received: 6 September 2018 / Published online: 17 October 2019

(c) The Author(s) 2019

\begin{abstract}
Finger joints in structural timber and glulam lamellae are often used to enable production of long members or to allow for re-connection of parts of a member after removal of weak sections. According to the European Standard EN 15497, certain margins are required between knots and a finger joint in structural timber, which means that a considerable amount of clear wood becomes waste when finger joints are applied. The purpose of this paper was to investigate the possibility of reducing the quantity of waste using different criteria for placement of finger joints. The investigation was based on (1) application of methods of colour scanning and tracheid effect scanning to detect knots and grain disturbance on board surfaces, and (2) interpretation of the requirements of EN 15497 regarding where finger joints may be placed. The standard's requirement when producing finger joints is that the minimum distance between a knot and a finger joint is three times the knot diameter. The standard allows for the minimum distance between a knot and a finger joint to be shortened to 1.5 times the diameter when the local fibre orientation is measured. Utilizing this in simulated production resulted in reduction of waste from 7.4 to $4.0 \%$, when using finger joints simply to produce timber of long lengths. If finger joints are also used to re-connect parts of members after removal of weak sections, even larger savings can be made. Furthermore, it is concluded that knowledge of fibre orientation obtained from scanning could be used not only to decrease the waste in production but also to increase the quality of finger joints.
\end{abstract}

\section{Introduction}

\subsection{Background}

The engineering properties of softwood timber vary considerably not only between species, but also within single species, between origins, stands, trees, boards and within boards. For example, the bending strength of boards of Norway spruce timber from Sweden, Norway and Finland may vary between $10 \mathrm{MPa}$ and $80 \mathrm{MPa}$ and the corresponding variation for modulus of elasticity $(\mathrm{MoE})$ is between 5 and $20 \mathrm{GPa}$ (Olsson and Oscarsson 2017). The shape stability of timber (timber distortion), when subjected to changes in

Anders Olsson

anders.olsson@lnu.se

Andreas Briggert

andreas.briggert@lnu.se

Jan Oscarsson

jan.oscarsson@lnu.se

1 Department of Building Technology, Linnaeus University, 35195 Växjö, Sweden moisture content, varies depending on the extent of spiral grain and on the part of the log cross section the board is cut from (Ormarsson 1999). Moreover, the length of logs restricts the length of boards that can be produced, unless finger joints are applied to give longer assembled members. All this means that production of structural timber with welldefined and appropriate engineering properties, and with length set by the needs of applications rather than from the length of the logs produced, requires both accurate grading with respect to engineering properties and application of finger joints. Competitiveness on the market also requires production at low costs. The latter can partially be achieved by minimizing the waste of material when applying finger joints.

The properties of structural timber of different strength classes are defined in the European standard EN 338 (2016). In this standard, characteristic values of several strength and stiffness properties are specified, in addition to characteristic density. However, machine strength grading of timber according to the European standard EN 14081-2 (2018) concerns only requirements on three different grade determining properties, namely the bending or tensile strength, the MoE 
in bending or tension and the density. In addition to these requirements, there are limitations regarding geometrical imperfections and visually identified defects, as specified in EN 14081-1 (2016). The yield achieved in different strength classes thus depends both on the quality of the timber and on the accuracy of the methods/machines used for prediction of the abovementioned grade determining properties. Regarding accuracy in grading it is, in most cases, the ability to predict strength rather than density or MoE, that limits the yield and, consequently, improved grading accuracy with respect to strength leads to higher yield in high strength classes (Olsson and Oscarsson 2017).

The most accurate machine strength grading methods available on the market are based on X-ray scanning or surface laser scanning, in combination with other techniques like dynamic excitation of longitudinal modes of vibration (Bacher 2008; Hanhijärvi and Ranta-Maunus 2008; Olsson and Oscarsson 2017). These methods also give the basis for assessment of the variation of strength along each board, such that a weak part of a board may be removed in order to enable grading of the remaining parts to a high strength class.

\subsection{Finger joints in structural timber}

Finger joints are applied to the production of structural timber and glulam lamellae mainly to produce long boards, but in some production lines also for the removal of weak/poor sections. Performance requirements and minimum production requirements of finger jointed solid/structural timber are given in the European standard EN 15497 (2014). Similar but not identical requirements for glulam laminations are given in EN 14080 (2013). Regarding the edgewise bending strength of finger joints, it is stated in EN 15497 that this strength shall be equal to or greater than the declared characteristic bending strength of the unjointed timber. Strength of finger joints depends on the wood properties but also on the geometry of the fingers, on the glue applied and on the skill of the manufacturer (Aicher 2003). The dependency between wood properties is such that higher clear wood MoEs, which correlate to higher strength of clear wood parallel to grain and higher shear strength, give stronger joints. These stiffness and strength properties correlate to the clear wood density and therefore it should be relatively easy to select timber that is well suited to give strong finger joints. Serrano (2000) performed finite element calculations on the influence of bond line properties and on the significance of defects in finger joints, and showed that even small defects in bond lines may have a considerable effect on the strength of a finger joint. The current situation regarding the edgewise bending strength of finger joints produced in industry in Sweden is that many manufacturers are certified to produce finger joints in structural timber strong enough for the strength class C30, some even for the class C35 (C30 and C35 refer to characteristic edgewise bending strength of $30 \mathrm{MPa}$ and $35 \mathrm{MPa}$, respectively) but not for higher C-classes (Ziethén 2017). Regarding finger joints in glulam lamellae (characterised by tensile strength classes, i.e. T-classes), the tensile strength of finger joints is indeed an issue, since failure on the tension side of glulam beams loaded in bending very often occurs in finger joints (Fink et al. 2015). Frese and Blass (2009) presented a research indicating that higher characteristic tensile strength of finger joints than required at the time is necessary in order to produce glued laminated timber (GLT) that meets the strength requirements. Current requirements on characteristic strength of finger joints for different GLT strength classes are specified in EN 14080.

Regarding production, one crucial requirement concerns a minimum distance between the finger joint and knots in the connected members, which must be long enough to ensure (1) that no significant grain deviation occurs within the finger-joint itself and (2) that the knot does not cause stress concentrations that reach into the finger joint. The minimum distance between the finger joint and a knot in structural timber is set (EN 15497) to three times the diameter of the knot (knots smaller than $6 \mathrm{~mm}$ may be disregarded) except where an appropriate automated system guarantees that in the range of the finger joint the grain orientation is parallel to the longitudinal direction of the board. In the latter case, the minimum distance to knots is set to 1.5 times the knot diameter, if the knot remains within one of the fingerjointed members. However, if the knot is removed there is no requirement on a distance of 1.5 times the knot diameter but only the requirement of straight fibres since, of course, a removed knot will not cause stress concentrations within the finger joint. Thus, control of size and location of knots and of local grain direction are required for the production of finger jointed structural timber. If these properties are not determined with sufficient accuracy, the consequence is that considerable margins must be added when removing sections with knots and/or fibre distortions to ensure that the requirements are not violated. This means, of course, waste of material. Neglect regarding sufficient margins, on the other hand, leads to violation of the standard and possibly to poor quality of finger joints which may result in failure of the joint itself.

The standard EN 15497 gives guidance regarding the interpretation of the knot diameter that defines the required minimum distance between a knot and the finger joint, but some additional interpretation is necessary. The drawing shown in Fig. 1a is resembling a drawing in EN 15497 and shows that for a round or oval knot the diameter, $d$, shall be understood as the width of the knot in the transversal direction of the board. The drawing in Fig. 1b shows an arris knot, and for such a knot, the diameter shall be set to the length of the knot in the longitudinal 
(a)

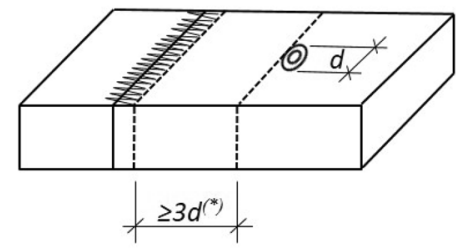

(b)

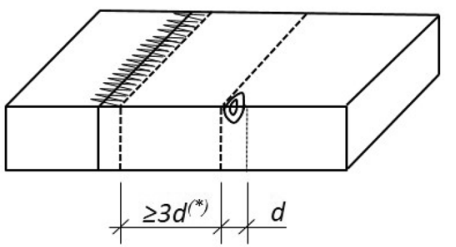

(c)

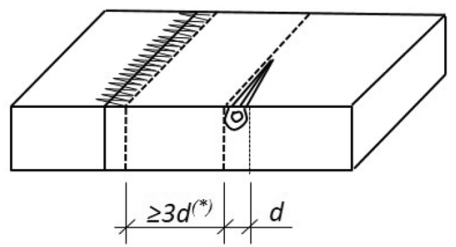

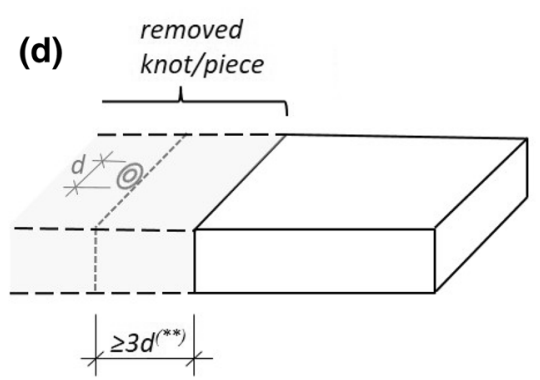

$\left(^{*}\right)$ alternative criterion: distance $\geq 1.5 d$ and straight fibers within finger joint

$\left(^{* *}\right)$ alternative criterion: straight fibers within finger joint
Fig. 1 Drawings of a finger jointed timber showing the minimum distance between knot and finger joint in cases where the knot is a a round or oval knot, $\mathbf{b}$ an arris knot, and $\mathbf{c}$ a splay knot, in each case remaining within the board to be finger-jointed, and $\mathbf{d}$ a case where the knot is removed. Definitions of knot diameter, $d$, for different types of knots illustrated are based on guidance given by EN 15497 and of interpretations thereof direction of the board. This definition is thus different from how knots are generally measured in visual grading (e.g. SS 230120 2010; Thunell 1981). Figure 1c shows a drawing of a splay knot. How to interpret knot diameter in this case is not explained in EN 15497, but the interpretation herein is to measure it in the same way as an arris knot, as indicated in Fig. 1c. Thus, in all cases where a knot is visible both on the wide and on the narrow edge, the knot diameter is interpreted as the length of the knot in longitudinal board direction, even if the size of the knot in transversal direction on one or more sides is larger. If a knot is removed, the requirements are somewhat different compared to if it remains within the timber. Figure 1d illustrates the case, and corresponding alternative margin requirements, when a knot is removed (the shaded part of the board will not be used).

A minimum distance between a knot (that remains in the member) and the finger joint as low as 1.5 times the knot diameter is, according to EN 15497, allowed between a knot and the finger joint if the grain orientation is parallel to the longitudinal direction of the board. However, EN 15497 does not specify tolerances for this requirement of fibres being parallel to the direction of the board but only expresses that there shall be no pronounced grain disturbance within the joints. This means that anyone who intends to implement this requirement in practice must interpret this requirement in measurable terms.

\subsection{Detection of knots on the basis of tracheid effect scanning}

When a softwood surface is illuminated with concentrated light, such as laser, fibres conduct light better in the direction of the fibres than across. This is referred to as the tracheid effect (Matthews and Beech 1976; Seltman 1992; Soest et al. 1993). The shape of a dot of light, which on a surface of an isotropic material would have been circular in shape, becomes, on a softwood surface, stretched out in the direction of the fibres and the major axis of the thus stretched, elliptically shaped spot indicates the local in-plane fibre direction. Attempts to detect knots on the basis of data from tracheid effect scanning have been presented both by Briggert et al. (2016) and by Kandler et al. (2016). In both studies, light dots on the wood surfaces from a laser source were assessed both with respect to the main direction of the elliptically shaped light spots, from which the in-plane fibre directions were obtained, and with respect to the roundness of the light spots. The latter was, in both investigations, assumed to give an indication of the so-called diving angle, i.e. the angle between the local fibre direction and the investigated surface. On the basis of this, 3D fibre directions were determined locally on wood surfaces with a resolution of about $4.4 \mathrm{~mm}$. A threshold was set for the angle between the determined local fibre direction and the longitudinal direction of the board such that positions on the wood surface where the determined angle exceeded a threshold of $56^{\circ}$ 
(Briggert et al. 2016) were regarded as being within a knot. In both studies (Briggert et al. 2016 and Kandler et al. 2016), knots were identified on surfaces with reasonable but not excellent accuracy with respect to size and shape of knots. The reasons for the limited accuracy being (1) the limited spatial resolution of the laser dot grid and (2) the uncertainty of the relationship between the roundness of the laser dots and the assessed diving angle.

\subsection{Aim and scope}

The aim of the present research is to investigate the potential yield of finger jointed machine strength graded timber. Since the accuracy regarding recognition of knots and fibre deviations, as well as how the requirements of EN 15497 are interpreted, are decisive for where finger joints may be applied, these issues are discussed in detail. More precisely, the following is included:

1. A procedure for determination of size and position of knots based on a combination of tracheid effect scanning and colour/light intensity scanning,

2. A discussion and interpretation of the EN 15497 requirements of distances between finger joints and knots, and of the requirement that fibres within the finger joint must be parallel to the longitudinal direction of the members,

3. Results in terms of calculated yield and waste for simulated production cases where finger joints are used simply to produce an infinitely long board, and another production case where finger joints are used to produce an infinitely long board in which weak sections are removed such that the produced board fulfils requirements for a certain strength class.

\subsection{Limitations}

The investigation is limited to planed boards of Norway spruce of size $45 \times 145 \mathrm{~mm}$ from Sweden, Norway and Finland. Other dimensions, and other species or origin, may give different results. Furthermore, results of the study are strictly valid only for structural timber, even though the results should give a relevant indication also for GLT laminations.

\section{Material and equipment}

\subsection{Sampling of material}

Sampling of the timber considered in the present research was first done to give the basis for a formal approval of a machine strength grading method described in Olsson et al. (2013) and Olsson and Oscarsson (2017), i.e. for fulfilment of requirements of initial type testing according to EN 14081-2 (2010+A1:2012), and for derivation of settings for a growth area covering Sweden, Norway and Finland. Thus, the sampling and the preparation of the timber, including drying aiming at $12 \%$ moisture content (MC), was performed in accordance with the requirements laid down in the standard. At the time, it was stated in EN 384 (2010), clause 5.2, that when assessing bending strength the critical section, i.e. the section along the board at which failure is expected to occur, shall be in a position that can be tested, that is within the loading heads in a four point bending test as described in EN 408 (2010+A1:2012). Therefore, boards having their critical section closer to one of the ends than about 6.5 times the board depth were discarded. Hence, in order to assess at least 900 pieces, required according to EN 14081-2 (2010+A1:2012), about 2100 pieces, ranging in size from 30 to $70 \mathrm{~mm}$ in thickness and from 70 to $245 \mathrm{~mm}$ in depth, were sampled at a first stage. Out of the 2100 pieces, 897 were of dimension $45 \times 145 \mathrm{~mm}$ and these constitute the sample for the present study. This latter sample was divided into five subsamples representing timber from (1) northern Sweden, (2) mid Sweden, (3) southern Sweden, (4) Norway, and (5) Finland. The numbers of specimens included in each region are presented in Table 1.

\subsection{Properties of investigated sample}

All the boards of the sample considered herein were assessed with respect to dimensions, weight, axial resonance frequency and tracheid effect scanning, as described in Olsson and Oscarsson (2017), but as explained above not all of them were actually subjected to destructive testing in four-point bending. Dynamic $\operatorname{MoE}\left(E_{\text {dyn }}\right)$ and board density $\left(\rho_{\text {corr }}\right)$, both adjusted to $12 \% \mathrm{MC}$ were, however, determined for all
Table 1 Number of specimens (all of dimension $45 \times 145 \mathrm{~mm}$ ) and length from each of the five subsamples/origins

\begin{tabular}{llll}
\hline Sub- sample & Origin & Length of specimens $(\mathrm{mm})$ & Number of specimens \\
\hline 1 & Sweden North & 4200 & 153 \\
2 & Sweden Mid & 4500 and 5100 & $154+140$ \\
3 & Sweden South & 3600 & 90 \\
4 & Norway & 4800 & 250 \\
5 & Finland & 4500 & 110 \\
& Total & & 897 \\
\hline
\end{tabular}


the boards of the present sample and mean values, standard deviations and coefficients of variation of these properties are given in Table 2. Given in Table 2 are also the mean MC of each subsample, determined on the basis of measurements of a limited number of boards of each sub sample using a pin type MC metre. Relationships between timber properties, in terms of coefficient of determination, are reported in Olsson and Oscarsson (2017).

\subsection{Equipment for scanning}

There are several different manufacturers of wood scanners operating on the market and scanning of sawn timber has been performed for different purposes at sawmills and in the wood working industry for more than 20 years. For the present research, an industrial scanner of make WoodEye (WoodEye 2018) was used. This machine is equipped with lasers and multi-sensor cameras, one set for each longitudinal surface of the board, for collecting data regarding the inplane fibre directions and high-resolution three colour photographs of the scanned surfaces. In the scanner, fibre direction data is obtained by means of the tracheid effect. Figure 2 shows (a) the industrial scanner used, (b) an enlarged photograph of a small wood surface, (c) the spread of light from a dot laser when illuminating the small wood surface, the drawn black line indicating the determined in-plane component of the fibre direction, (d) an image/photograph obtained from the scanner showing a larger wood surface, and (e) the determined local in-plane component of the fibre directions over the same surface represented by small black lines. Within knots, such as the two displayed in Fig. 2d, fibres are actually directed almost perpendicular to the surface displayed. Thus, the in-plane component of the fibre direction is very small in such areas and laser light spots become almost circular in shape. As a result, the determined in-plane component of the fibre directions are uncertain within the knot areas marked by shadowed fields in Fig. 2e.

Data corresponding to what is displayed for one wood surface in Fig. 2d, e can be collected by a scanner for all four sides and for the full length of a wooden board when scanned at a speed as high as $450 \mathrm{~m} / \mathrm{min}$, which is a common operation speed in industry. The resolution of the fibre direction data obtained in the transverse direction of the board surfaces is $4.4 \mathrm{~mm}$ (resolution depends on the beam
Table 2 Dynamic MoE and board density, both corrected with respect to $\mathrm{MC}$, and $\mathrm{MC}$ determined using a pin type $\mathrm{MC}$ metre

\begin{tabular}{|c|c|c|c|c|c|c|c|}
\hline \multirow[t]{2}{*}{$\begin{array}{l}\text { Sub-sample } \\
\text {. }\end{array}$} & \multicolumn{3}{|c|}{$E_{\mathrm{dyn}}\left[\mathrm{N} / \mathrm{mm}^{2}\right]$} & \multicolumn{3}{|c|}{$\rho_{\text {corr }}\left[\mathrm{kg} / \mathrm{m}^{3}\right]$} & \multirow{2}{*}{$\begin{array}{l}\mathrm{MC}[\%] \\
\mathrm{m}\end{array}$} \\
\hline & $\mathrm{m}$ & $\mathrm{s}$ & $\mathrm{CoV}$ & $\mathrm{m}$ & $\mathrm{s}$ & $\mathrm{CoV}$ & \\
\hline 1 & 13300 & 1960 & 0.15 & 467 & 36 & 0.08 & 17.0 \\
\hline 2 & 12200 & 1660 & 0.14 & 435 & 38 & 0.09 & 14.5 \\
\hline 3 & 13400 & 2450 & 0.18 & 480 & 39 & 0.08 & 19.3 \\
\hline 4 & 12900 & 2160 & 0.17 & 472 & 57 & 0.12 & 13.5 \\
\hline 5 & 12700 & 1910 & 0.15 & 456 & 40 & 0.09 & 13.6 \\
\hline All & 12800 & 2020 & 0.16 & 458 & 47 & 0.10 & 15.0 \\
\hline
\end{tabular}

$m$ mean value, $s$ standard deviation, $\mathrm{CoV}$ coefficient of variation
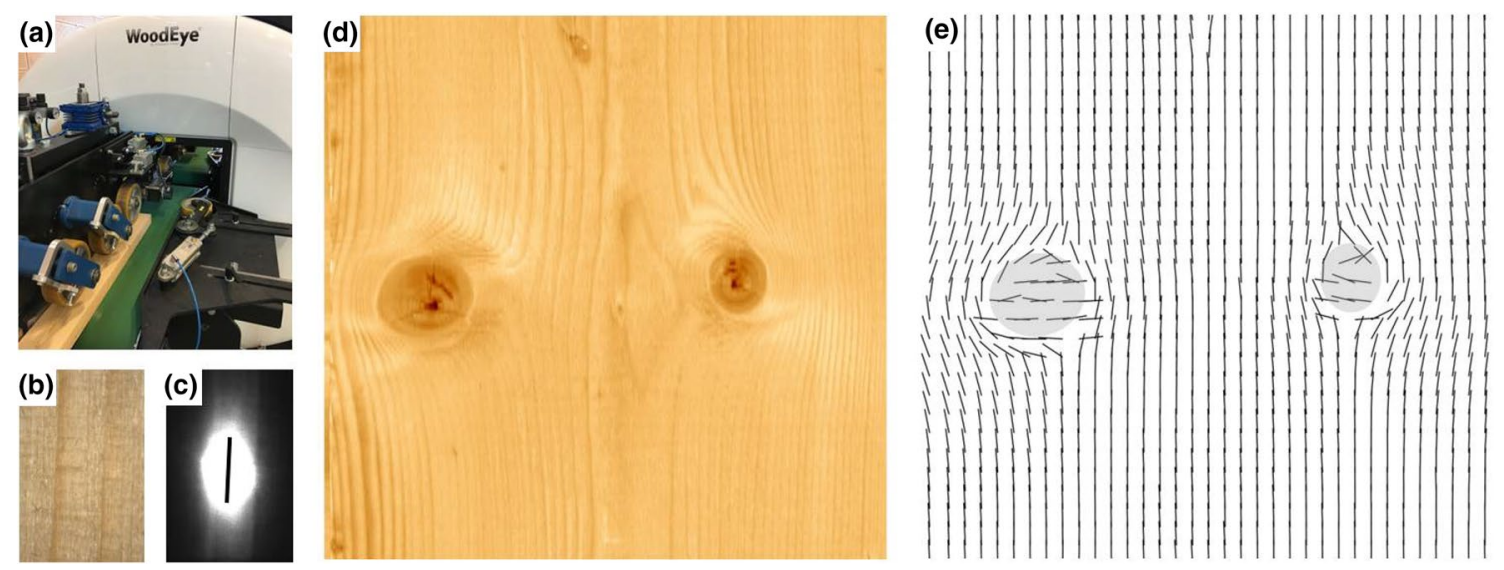

Fig. 2 a Industrial scanner used, b enlarged photograph of a small wood surface, $\mathbf{c}$ spread of light from a dot laser illuminating the small wood surface, drawn black line indicating the in-plane fibre direction determined on the basis of the shape of the spread of light, $\mathbf{d}$ image obtained from the scanner showing a wood surface and e local inplane fibre directions of the surface shown in $\mathbf{d}$ determined by the scanner, two shaded fields indicating areas where the in-plane component of the fibre direction is uncertain 
diffraction splitter installed on the dot laser source) and with a conveyor speed of $450 \mathrm{~m} / \mathrm{min}$, the same resolution is obtained in longitudinal direction. Regarding colour photographs, the data obtained consists of three colours, red, blue and green, and the resolution is as high as $0.07 \mathrm{~mm}$ in the transverse direction of the board and $0.8 \mathrm{~mm}$ in longitudinal board direction. Furthermore, by means of the scanning operation, it is also possible to determine length, thickness and depth of the investigated boards.

\section{Methods and implementation}

\subsection{Detection of weak sections along boards}

The machine strength grading method mentioned in Sect. 2.1, Sampling of material, was used to calculate bending MoE profiles for each board. Such profiles give the basis to decide which sections to remove by cross-cut, in order to produce finger-jointed structural timber of certain quality. In addition to data from tracheid effect scanning, the method to calculate bending MoE profiles utilizes knowledge of longitudinal resonance frequency and weight obtained by a complementary strength grading machine. In brief, the method to calculate MoE profiles comprises the following stages (for further details see Olsson et al. 2013; Olsson and Oscarsson 2017):

- Nominal values of nine wood material parameters (MoEs in $l$-, $r$, and $t$-direction $E_{1,0}, E_{\mathrm{r}, 0}$ and $E_{\mathrm{t}, 0}$, respectively, shear modulus in three orthogonal planes, $G_{1 \mathrm{r}, 0}, G_{\mathrm{lt}, 0}$ and $G_{\mathrm{rt}, 0}$, and Poisson's ratios $v_{\mathrm{lr}}, v_{\mathrm{lt}}$ and $\left.v_{\mathrm{rt}}\right)$ are employed, as shown in Table 3.

- For each position on the surfaces of the board where a local fibre orientation is determined, the MoE in the longitudinal (x-) direction of the board, $E_{\mathrm{x}}$, is calculated as a function of the nominal material parameters and the locally determined fibre orientation. As a result, in positions where the local fibre orientation deviates substantially from the longitudinal direction of the board,

Table 3 Nominal material parameters employed (Norway spruce), values originating from Dinwoodie (2000)

\begin{tabular}{ll}
\hline$E_{1,0}$ & $10,700 \mathrm{MPa}$ \\
$E_{\mathrm{r}, 0}$ & $710 \mathrm{MPa}$ \\
$E_{\mathrm{t}, 0}$ & $430 \mathrm{MPa}$ \\
$G_{\mathrm{lr}, 0}$ & $500 \mathrm{MPa}$ \\
$G_{\mathrm{lt}, 0}$ & $620 \mathrm{MPa}$ \\
$G_{\mathrm{rt}, 0}$ & $24 \mathrm{MPa}$ \\
$v_{\mathrm{lr}}$ & 0.38 \\
$v_{\mathrm{lt}}$ & 0.51 \\
$v_{\mathrm{rt}}$ & 0.51 \\
\hline
\end{tabular}

$E_{\mathrm{x}}$ becomes quite low, since wood is weak in directions perpendicular to grain.

- On the basis of calculated $E_{\mathrm{x}}$ as a function of the position over the surfaces of the board, and assumptions for $E_{\mathrm{x}}$ of the interior of the board, an MoE valid for edgewise bending is calculated by integration over the board cross section. Thus, a bending stiffness profile representing the board is obtained and the lowest value along the profile is used as an indicating property (IP) to bending strength.

- Knowledge of the axial resonance frequency and weight of the board is used to calibrate the bending stiffness profile since the material parameters, for which nominal values are displayed in Table 3, are actually different for each board.

Figure 3 gives an illustration of the method showing (a) local fibre directions scanned on a board surface, (b) board cross-section divided into sub-areas implying that the exhibited angle $\varphi$ and corresponding MoE in the longitudinal direction, $E_{\mathrm{x}}(x, y, z)$, to be valid within the volume $\mathrm{dA} \times \mathrm{dx}$, (c) segment of length $d x$ for which the edgewise bending MoE is calculated by stiffness integration over the segment's cross-section, and (d) a bending MoE profile, each value along the graph representing the average edgewise bending $\mathrm{MoE}$ of the surrounding $90 \mathrm{~mm}$, and the lowest value along the profile defining the IP to bending strength.

Obtained bending MoE profile, such as the one shown in Fig. 3d, indicates where along each board assessed, the calculated bending stiffness is at its lowest. This is utilized for the prediction of the strength of the board. In the present investigation, the information is utilized in simulated production of finger jointed machine strength graded structural timber.

Some other strength grading methods/machines, for example those based on X-ray technology, enable lengthwise resolution of predicted bending strength in a similar way and there are sawmills utilizing this feature of X-ray based machines to eliminate weak section and reconnect, by finger joints, the remaining parts. When this is utilized, the assembled members are assigned to higher strength classes than what would be allowed if the weak sections of the original, full-length member were not removed (Ziethén 2017).

\subsection{Detection of knots}

The method developed by Briggert et al. (2016) for the detection of knots on wood surfaces on the basis of tracheid effect scanning, as briefly described in Sect. 1.3, was utilized in a first step for the detection of knots in the boards of the present sample. Then, a rectangular area, enclosing the knot surface, was determined by adding margins of $3 \mathrm{~mm}$ on each side of the knot in transversal direction of the board and of $5 \mathrm{~mm}$ on each side in longitudinal direction. Figure 4 
(a)

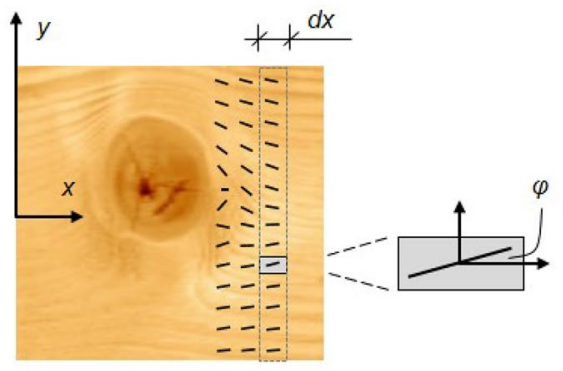

(b)

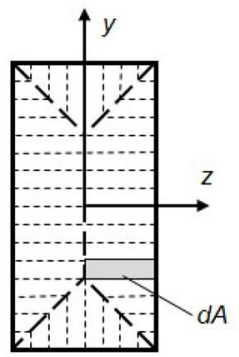

(c)

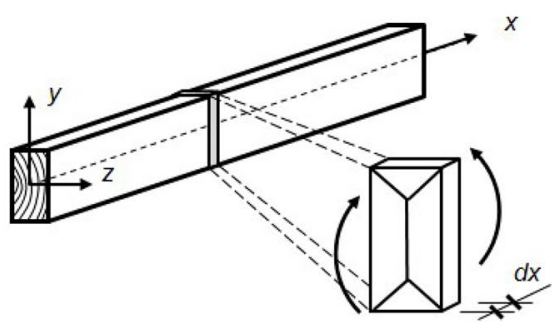

(d)

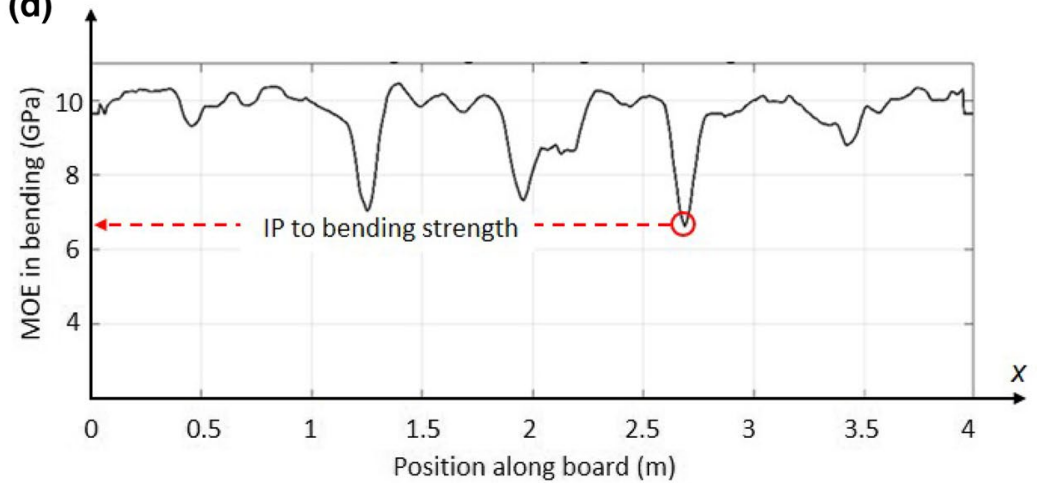

Fig. 3 a Local fibre directions scanned on a member's surface by means of a row of laser dots, $\mathbf{b}$ cross-section divided into sub-areas implying that the exhibited angle $\varphi$ and corresponding MoE in the longitudinal direction, $E_{\mathrm{x}}(x, y, z)$, is valid within the volume $\mathrm{dA} \times \mathrm{dx}$, c segment of length $d x$ for which the edgewise bending MoE is cal-

shows photographs/images and local fibre directions, both obtained by a scanner, on four wood surfaces of a $250 \mathrm{~mm}$ long section of a timber member, $45 \times 145 \mathrm{~mm}$ in size. The drawn red rectangles mark the abovementioned rectangular knot containing areas.

Within the identified rectangular areas, knots were then identified again but now on the basis of the light intensity of the images of the wood surfaces. Actually, the only image utilized, out of the three available, was the red scaled one, although the blue scaled or green scaled images could have been utilized as well. For each pixel of the image a number between 0 (very dark) and 255 (very bright) is obtained and a threshold was set below which the pixel is considered dark enough to be part of a knot. The threshold was, however, not a fixed number but adjusted in relation to the brightness of a surrounding, semi-local area in the following way: At a given longitudinal position $p$ on a board surface, the median value, denoted $m_{\mathrm{e}}$, of all light intensity values of a line of pixels oriented in transversal board direction was determined. Then the median value of all such median values of lines within a range of $300 \mathrm{~mm}$ in longitudinal board direction was determined. This median value was denoted $n_{\mathrm{p}}$, and the dynamic threshold to be used for a pixel in the current longitudinal position, $p$, was calculated as culated by stiffness integration over the segment's cross-section, and d a bending MoE profile, each value along the graph representing the average edgewise bending $\mathrm{MoE}$ of the surrounding $90 \mathrm{~mm}$, and the lowest value along the profile defining the IP to bending strength

$t_{\mathrm{p}}=\frac{t_{\mathrm{s}}}{256} n_{\mathrm{p}}$

where $t_{\mathrm{s}}$ is a fixed number, here set to 200 based on trial and error, and comparisons of results and images made with the naked eye. Thus, if the light intensity number of the pixel considered (a number between 0 and 255) is lower than $t_{\mathrm{p}}$ then this pixel/position indicates knot surface. Considering the pixels contained within an investigated rectangular area (red rectangles in Fig. 4) one or several coherent group/groups of knot indicating pixels are usually identified, although sometimes no knot indicating pixels are contained within the area. Convex hulls that encircle such coherent groups of knot indicating pixels are determined and the largest one within each rectangular area is regarded a knot. Knots identified in this way are encircled by cyan blue contours in Fig. 4. The reason to look for knots only within the rectangular areas is that this reduces the risk of mistaking, for example, dirt on the surface for a knot.

Surface (d) of Fig. 4 is located close to the pith of the log from which the piece of timber is cut (the pith is located at a distance of only 5-10 $\mathrm{mm}$ from this surface, outside the piece). On such surface, the borders of the knots are often hard to distinguish on the basis of surface darkness, i.e. the 


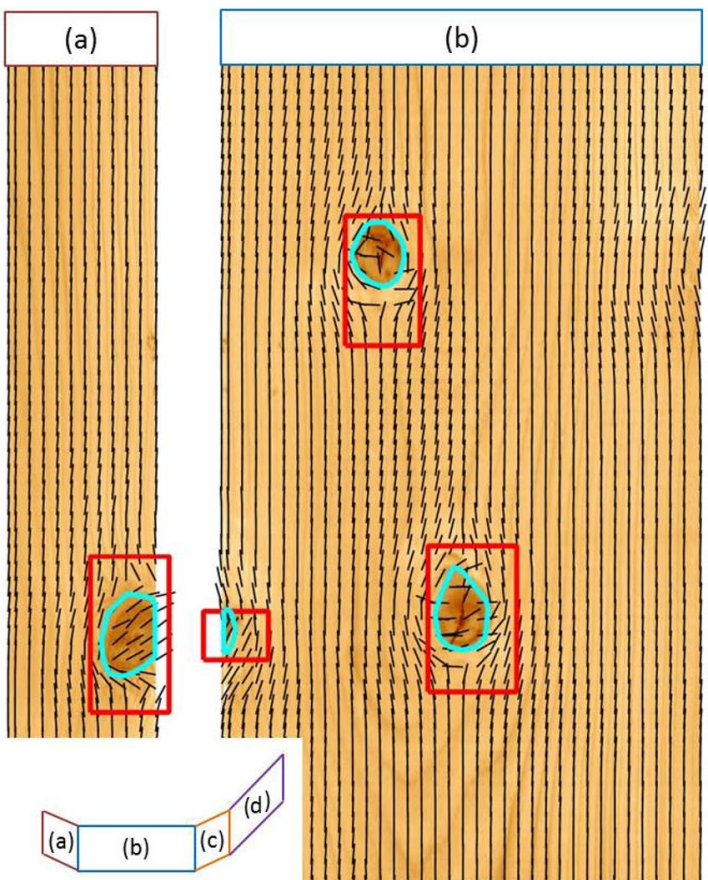

Fig. 4 Four wood surfaces (a-d) of one piece of timber. Each red rectangle, determined on the basis of tracheid effect scanning, contains a knot. These, encircled in cyan blue, are determined on the basis of optic scanning and a threshold of light intensity. Small black lines

identification is sensitive to the threshold set by Eq. 1, and in this particular case it is quite clear that the size of the knot at the top left on this surface is underestimated. The knots to the top right and to the bottom left on surface (d) are traversing knots that are also visible as larger round knots on surface (b). Likewise, the knots to the top left and to the bottom right on surface (d) are also visible on surface (c) and (a), respectively. All the knots visible on surfaces (a-c) seem to be accurately determined. A small part of the knot visible on surface (a) is also identified on surface (b). Hence this is an example of an arris knot for which the knot diameter is calculated as the length of the knot in longitudinal direction of the board, here determined on surfaces (a), as explained in Sect. 1.2. Further comments on the results of the described knot detection procedure, of which Fig. 4 shows just an example, are given in Sect. 4 below. Finally, a knot diameter, $d$, is determined for each detected knot as described in Sect. 1.2 above.

\subsection{Criteria of pronounced grain disturbance}

To determine whether there is any pronounced grain disturbance in a certain section along the board, i.e. at a certain position in longitudinal direction, the following is considered. For the position $p$ in longitudinal direction (position in millimetre from one end of the board), the fibre directions
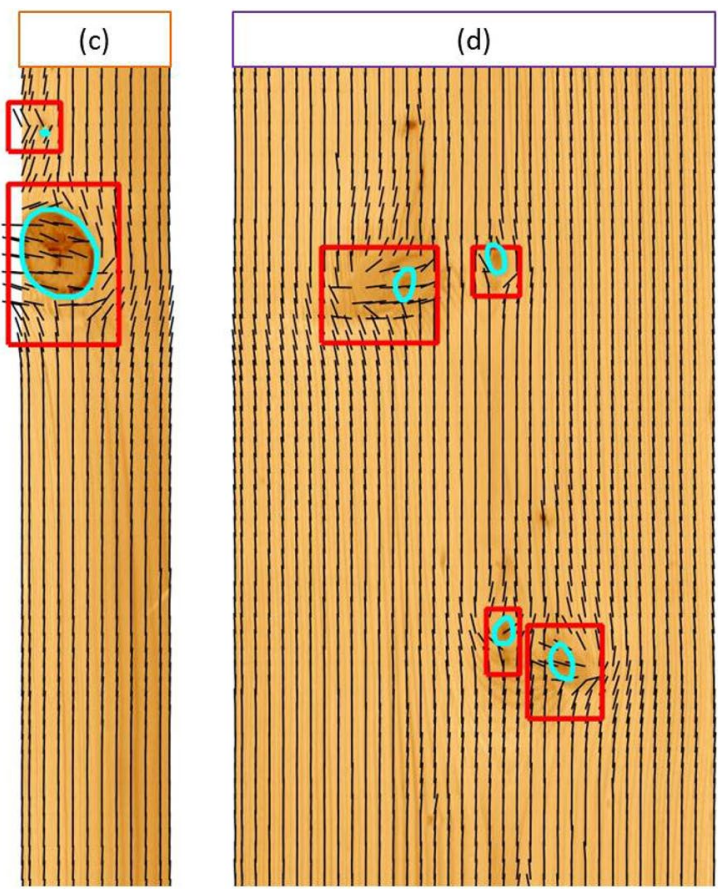

indicate local in-plane fibre directions, determined on the basis of the tracheid effect. The drawing added at the bottom left illustrates how the four sides $(\mathrm{a}-\mathrm{d})$ of the same piece are related to each other

of the scanning grid, on all four sides and within the section $p \pm 5 \mathrm{~mm}$, is considered. This is done for all $10 \mathrm{~mm}$ long sections along the board, i.e. for $p=5,15,25, \ldots, L-5 \mathrm{~mm}$, where $L$ is the length of the board. Then, each $10 \mathrm{~mm} \mathrm{sec-}$ tion along the board is regarded as a section with or without pronounced grain deviation on the basis of a criterion. Four different criteria are considered herein to define a section with pronounced grain disturbance, namely the following:

(i) more than $10 \%$ of all the determined fibre directions within the $10 \mathrm{~mm}$ long section exceed a grain deviation of $8^{\circ}$,

(ii) more than $10 \%$ of all the determined fibre directions within the $10 \mathrm{~mm}$ long section exceed a grain deviation of $8^{\circ}$ or more than $5 \%$ of all the determined fibre directions within the $10 \mathrm{~mm}$ long section exceed a grain deviation of $13^{\circ}$,

(iii) more than $5 \%$ of all the determined fibre directions within the $10 \mathrm{~mm}$ long section exceed a grain deviation of $13^{\circ}$.

(iv) more than $10 \%$ of all the determined fibre directions within the $10 \mathrm{~mm}$ long section exceed a grain deviation of $13^{\circ}$.

The thresholds used for grain deviation in criteria (i-iv) are set based on the following considerations. A simple 
expression for the tensile strength as a function of fibre angle is given by Hankinson's formula (Hankinson 1921) as

$$
f_{t, \alpha}=\frac{f_{t, 0} f_{t, 90}}{f_{t, 0} \sin ^{n} \alpha+f_{t, 90} \cos ^{n} \alpha}
$$

where $f_{t, 0}$ and $f_{t, 90}$ are tensile strength along and perpendicular to the fibre direction, respectively, $n$ is a parameter to be set empirically on the basis of experimental results and $\alpha$ is the fibre angle, i.e. the grain deviation. Using a relationship of $f_{t, 0} / f_{t, 90}=30$ and $n=1.7$, which is representative for what has been reported in literature for Norway spruce, although different investigation have shown different results (Gustafsson 2003), a grain deviation of $8^{\circ}$ means a reduction in tensile strength of about $50 \%$. Correspondingly, a grain deviation of $13^{\circ}$ means a reduction in tensile strength of about $70 \%$. This means, of course, that grain deviation smaller than $8^{\circ}$ may have considerable influence on the strength of finger joints but for reasons discussed in Sect. 4.2, a smaller grain deviation than $8^{\circ}$ is neglected herein.

\subsection{Criteria for placement of finger joints}

Detection and definition of knots and pronounced grain disturbance give the basis for the definition of the criteria concerning where along the boards finger joints may, or may not, be placed. Eight different criteria are considered herein, namely:

1. Finger joints may not be placed closer to a knot than three times the diameter of the knot (knot diameters are defined in Sects. 1.2 and 3.2).

2. Finger joints may not be placed closer to a knot than 1.5 times the diameter of the knot.

3. Finger joints may not be placed within, or closer than $10 \mathrm{~mm}$ to, sections that contain pronounced grain disturbance according to criterion i (no more than $10 \%$ grain exceeding $8^{\circ}$ ), see Sect. 3.3.

4. Finger joints may not be placed within, or closer than $10 \mathrm{~mm}$ to, sections that contain pronounced grain disturbance according to criterion ii (no more than $10 \%$ grain exceeding $8^{\circ}$ or more than $5 \%$ grain exceeding $8^{\circ}$ ).

5. Finger joints may not be placed within, or closer than $10 \mathrm{~mm}$ to, sections that contain pronounced grain disturbance according to criterion iii (no more than $5 \%$ grain exceeding $13^{\circ}$ ).

6. Finger joints may not be placed within, or closer than $10 \mathrm{~mm}$ to, sections that contain pronounced grain disturbance according to criterion iv (no more than $10 \%$ grain exceeding $13^{\circ}$ ).

7. Combination of criteria (2) and (4) to represent one of the two alternatives of EN 15497 regarding minimum distance, i.e. none of these two criteria may be violated in the case when the knot remains within the finger jointed timber, and criterion (4) may not be violated when the knot is removed. The notation $((2 \cup 4) / 4)$ is used below for this case.

8. Intersection of criteria (1) and (7), i.e. for each section along a board it is sufficient if one of these two criteria is fulfilled. The notation $(1 \cap 7)$ is used below for this case.

Of course, the length of a section where a finger joint may be applied must always be at least as long as the length of the finger joint itself. Herein the length of a finger joint is set to $30 \mathrm{~mm}$. Criteria (1), (7) and (8) could, according to the author's interpretation of EN 15497, be used in the production, whereas the other criteria cannot.

\subsection{Simulated production cases}

Two different cases of production of finger jointed sawn timber are simulated herein. These are

(a) Production of finger jointed timber without any requirements on strength class of the members to be connected, i.e. the only requirement applied concerns where finger joints are allowed, i.e. criteria (1-8) of Sect. 3.4.

(b) Production of finger jointed timber of strength class C35. The grading method utilized is the method of Olsson and Oscarsson (2017) with settings for the class $\mathrm{C} 35$, valid for single grade. In this production case, sections along the boards for which the calculated bending MoE is below $9.84 \mathrm{GPa}$ (boards with such sections may not be graded in full length to the class C35) are removed and the remaining, sound parts are reconnected by finger joints, with application of criteria 1-8 of Sect. 3.4.

For all cases it is required that the length of utilized members are at least $500 \mathrm{~mm}$ long. Shorter members than this are always discarded. For both production cases (a) and (b), the principle is that a single infinitely long board, consisting of boards connected by finger joints according to one of the eight criteria defined in Sect. 3.4, is produced.

\section{Results and discussion}

\subsection{Observations regarding detection of knots}

Detection of knots as described in Sect. 3.2 gives, according to visual inspection, i.e. manual inspection of results represented by images such as those displayed in Fig. 4, of a high number of boards, accurate results. This holds for the 
vast majority of boards and knots and the example of wood surfaces including knots shown in Fig. 4 is representative for the knot identification performed herein. However, as for the example shown in Fig. 4d, the size of surfaces of splay knots, which are common on wood surfaces close to the pith of boards, and usually brighter in colour than what other knots are, is often underestimated. Since such knot surfaces may be disregarded with respect to the knot diameter defined in Sect. 1.2 this is, however, not an issue for the applications considered in this research. For other applications, where it may be important to identify splay knots accurately, additional use can be made of the fibre directions identified on wood surfaces using the tracheid effect, since the fibre direction within splay knots coincides with the direction of the knot itself.

Even apart from splay knots, the knot detection algorithm presented herein fails in a few cases. For example, this can happen if an area that actually contains more than one knot is identified as one coherent fibre distorted area (fibre distorted areas are marked by red rectangles in Fig. 4). In such a case, two different misinterpretations can be made. One is that only the biggest knot within the fibre distorted field is identified, whereas smaller knots that are also contained within the area are erroneously disregarded, or that two or more smaller knots within the area are identified as a single large knot. However, finger joints are not seriously misplaced because of such misinterpretations.

\subsection{Observations regarding pronounced grain disturbance}

The identification of pronounced grain disturbance, as defined in Sect. 3.3 and used in finger joint criteria (3-8), see Sect. 3.4, is even more reliable than the detection of knots. Unless the wood surface is rough or very dirty the fibre directions identified on the basis of the tracheid effect are reliable, i.e. measurement errors are negligible compared to the comparatively large fibre angles that indicate pronounced grain disturbance. Errors caused by rough or very dirty wood surfaces could lead to identification of pronounced grain disturbance where, in reality, no such disturbance would occur on the corresponding well-planed surface, but such cases have not been observed in this study. The opposite situation, that fibres are identified as straight where they are actually disturbed, can be ruled out. Thus, any incorrect identification caused by rough or very dirty wood surface would lead to errors on 'the safe side' with respect to where finger joints are placed. It should be noted, however, that the present study is performed on planed timber.

A small number of the boards included in the sample were probably cut from non-straight logs or from logs with considerable spiral grain. For a study on the occurrence and magnitude of spiral grain in Norway spruce, see Säll
(2002). As a result, for about 3\% of the boards, the inclination of fibres observed on large parts of the wood surfaces amounts to more than $6^{\circ}$ also where the fibre orientation is not affected of knots. Therefore, fibre deviations below $8^{\circ}$ are not considered herein as pronounced grain disturbance, even if fibre deviations of 6-8 degrees correspond to a significant reduction in strength. It can be questioned, of course, if boards with such inclination of fibres should be used at all for the production of finger jointed structural timber. However, using finger joint criterion (1), which is based solely on the distance to knots, no boards would be discarded because of general inclination of fibres and therefore, in order to make a fair comparison of the yield using different criteria, the lowest threshold employed regarding fibre deviation was set to $8^{\circ}$.

\subsection{Application of criteria for finger joints}

Figure 5 shows (a) a calculated bending MoE profile of one board where red lines indicate positions/sections where the calculated bending MoE, see Sect. 3.1, is lower than what is required for strength class C35 (Olsson and Oscarsson 2017), (b) photographs of four sides of the board where coloured horizontally drawn lines indicate longitudinal sections that would pass for class C35 (green lines), sections that would not pass as C35 (red lines), sections that contain pronounced grain disturbance according to criterion (4) (black lines), sections with a knot present within a distance in longitudinal direction shorter than 1.5 times the knot diameter (blue lines), and sections with a knot present within a distance shorter than 3 times the knot diameter (purple lines). Thus, purple lines correspond to finger joint criterion (1) and blue lines correspond to criterion (2). In the case when the knot remains in the timber, the union of blue and black lines corresponds to criterion (7), whereas in the case when the knot is removed, black lines alone correspond to criterion (7). Figure $5 \mathrm{c}$ shows enlarged images of a $600 \mathrm{~mm}$ long part of the board. The vertically drawn purple arrows indicate where the board would be cut if criterion (1) was used to remove the weak section represented by the red marked field, as is done in production cases (b) defined in Sect. 3.4, i.e. the wood between the two purple arrows would then have to be discarded. The black arrows indicate where the board would be cut if criterion (7) was used instead. In the case illustrated in Fig. 5c there is a short gap in the horizontally drawn purple line between the two purple arrows but since this gap is shorter than $30 \mathrm{~mm}$, which is the assumed length of a finger joint, a finger joint cannot be placed there.

Figure 5 illustrates a typical case showing that finger joint criterion (1) gives, in general, longer continuous sections along which finger joints may not be placed, compared to what, for example, criterion (7) does. Thus, the use of 
Fig. 5 a Calculated bending MoE profile of a board, red lines indicating positions where the bending MoE is lower than what is required for strength class $\mathbf{C} 35$, b photographs of four sides of the member, coloured lines indicating longitudinal positions that would pass for class C35 (green lines), that would not pass as C35 (red lines), with significant fibre distortions according to criterion (4) (black lines), with a knot present within a distance shorter than 1.5 times the knot diameter (blue lines), with a knot present within a distance shorter than 3 times the knot diameter (purple lines), c enlarged image of a $600 \mathrm{~mm}$ long part of the board where dashed black and purple arrows indicate where the board would be cut, according to two different finger joint criteria, to remove a piece of timber not passing as $\mathrm{C} 35$
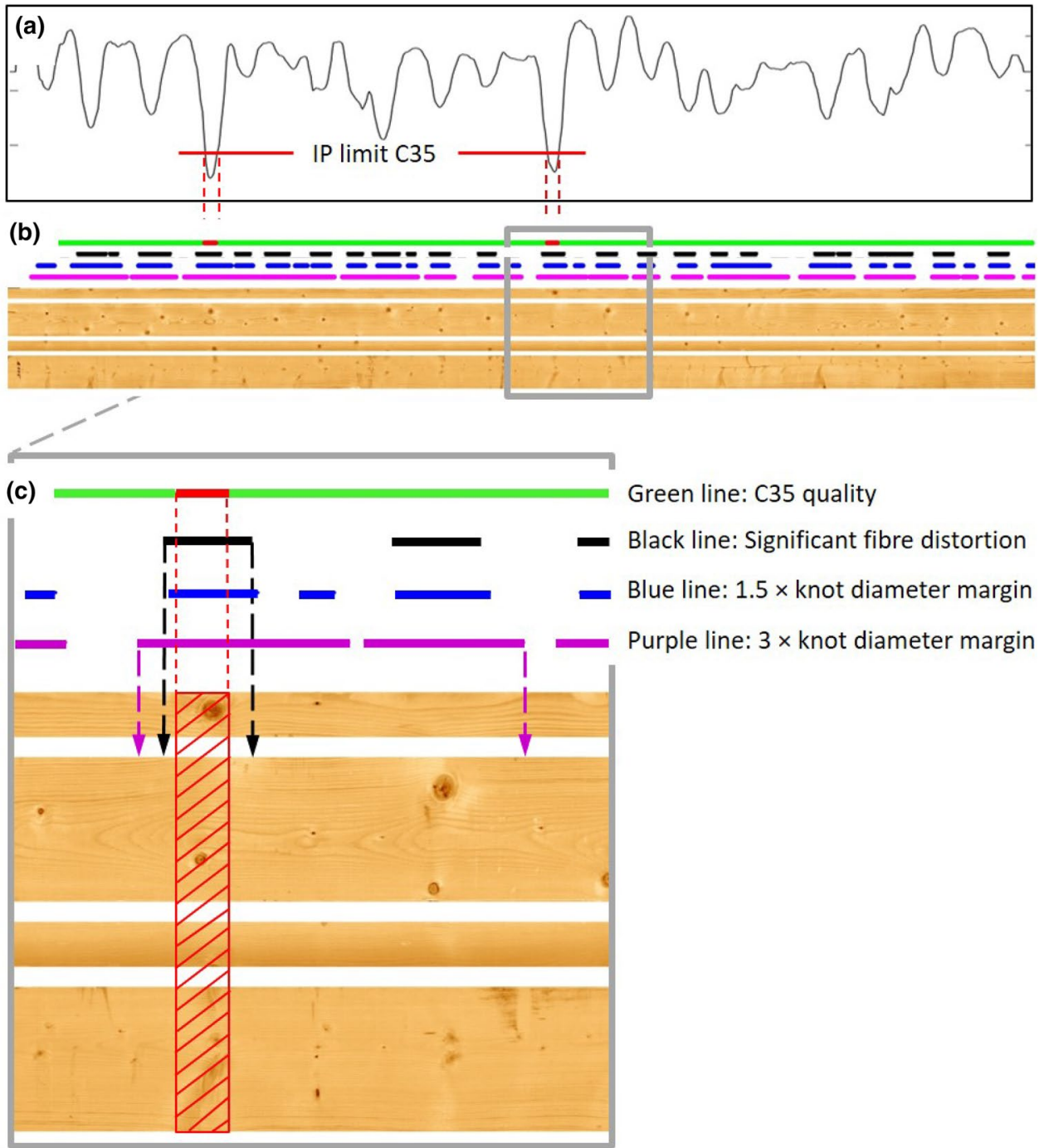

criterion (1) should, in most cases, result in longer sections discarded than what the use of criterion (7) would.

\subsection{Calculated yield of simulated production cases}

Table 4 shows the calculated percentage of waste of the total volume of the timber used to simulate production of fingerjointed timber, for each of the eight different finger joint criteria defined in Sect. 3.4 and for the two different production cases defined in Sect. 3.5. It is shown both for the entire sample and separately for each sub-sample. The yield percentage of the simulated production is equal to $100 \%$ minus the percentage of waste. Focusing first on production case (a) and the entire sample of boards including all regions, and comparison of criteria (1) and (2), it is clear that a minimum distance between a knot and a finger joint of 1.5 times the knot diameter, rather than of three times the knot diameter, would reduce the waste from about 7.4 to $3.1 \%$. However, criterion 2 is not permitted according to EN 15497, unless it is supplemented by or (if the knot is removed) replaced by a requirement of more or less straight fibres within the finger joints. Criterion (7) represents such a composite criterion and, for this, the calculated waste was $4.0 \%$, which is $3.4 \%$ unit points lower compared to when criterion (1) is used. Note that the use of criterion (1), where only the presence of knots is taken into account, means that there is still a risk that the finger joint will contain pronounced grain disturbance. This will certainly be the case, for example, when a large knot is cut off just outside the board's end before the board is connected to another one. For this reason, criterion (1) may, for some finger joints, give less waste than what criterion (7) does. Producers could use criterion (1), whenever it gives the least waste, and criterion (7), whenever it gives the least waste. Such a procedure is represented by criterion (8), which gives less than half of the waste compared to using criterion (1). Criterion (4), which is part of criteria (7) and (8), represents one possible interpretation of the requirement of EN 15497 regarding unacceptable fibre distortion 
Table 4 Calculated percentage of waste of the entire sample, and for each of the subsamples separately, for seven different finger joint criteria and for two different simulated production cases

\begin{tabular}{|c|c|c|c|c|c|c|}
\hline \multirow[t]{3}{*}{ Finger joint criterion } & \multicolumn{2}{|c|}{$\begin{array}{l}\text { All regions } \\
897 \text { boards }\end{array}$} & \multicolumn{2}{|c|}{$\begin{array}{l}\text { Northern Sweden } \\
153 \text { boards }\end{array}$} & \multicolumn{2}{|c|}{$\begin{array}{l}\text { Mid Sweden } \\
294 \text { boards }\end{array}$} \\
\hline & \multicolumn{2}{|c|}{ Production case } & \multicolumn{2}{|c|}{ Production case } & \multicolumn{2}{|c|}{ Production case } \\
\hline & $\begin{array}{l}\text { (a), i.e. } \\
{[\%]}\end{array}$ & (b), i.e. C35 [\%] & (a) $[\%]$ & (b) $[\%]$ & (a) $[\%]$ & (b) $[\%]$ \\
\hline $1(3 \phi)^{\mathrm{a}}$ & 7.4 & 28.1 & 9.8 & 21.9 & 6.1 & 26.5 \\
\hline $2(1.5 \phi)^{\mathrm{b}}$ & 3.1 & 21.2 & 4.0 & 15.0 & 2.7 & 20.1 \\
\hline $3\left(10 \% 8^{\circ}\right)^{b}$ & 3.1 & 20.8 & 4.3 & 15.3 & 2.1 & 19.1 \\
\hline $4\left(10 \% 8^{\circ} \cup 5 \% 13^{\circ}\right)^{\mathrm{b}}$ & 3.3 & 21.1 & 4.6 & 15.4 & 2.3 & 19.3 \\
\hline $5\left(5 \% 13^{\circ}\right)^{\mathrm{b}}$ & 2.2 & 19.6 & 2.7 & 13.5 & 1.7 & 18.6 \\
\hline $6\left(10 \% 13^{\circ}\right)^{\mathrm{b}}$ & 1.6 & 18.6 & 1.8 & 12.5 & 1.3 & 17.9 \\
\hline $7((2 \cup 4) / 4)^{\mathrm{a}}$ & 4.0 & 21.7 & 5.4 & 16.2 & 3.0 & 19.8 \\
\hline $8(1 \cap 7)^{\mathrm{a}}$ & 3.4 & 21.3 & 4.3 & 15.4 & 2.7 & 19.7 \\
\hline \multirow[t]{3}{*}{ Finger joint criterion } & \multicolumn{2}{|c|}{$\begin{array}{l}\text { Southern Sweden } \\
90 \text { boards }\end{array}$} & \multicolumn{2}{|c|}{$\begin{array}{l}\text { Norway } \\
250 \text { boards }\end{array}$} & \multicolumn{2}{|c|}{$\begin{array}{l}\text { Finland } \\
110 \text { boards }\end{array}$} \\
\hline & \multicolumn{2}{|c|}{ Production case } & \multicolumn{2}{|c|}{ Production case } & \multicolumn{2}{|c|}{ Production case } \\
\hline & (a) $[\%]$ & (b) $[\%]$ & (a) $[\%]$ & (b) $[\%]$ & (a) $[\%]$ & (b) $[\%]$ \\
\hline $1(3 \phi)^{\mathrm{a}}$ & 8.1 & 28.4 & 8.0 & 33.5 & 6.3 & 27.7 \\
\hline $2(1.5 \phi)^{\mathrm{b}}$ & 4.0 & 21.7 & 3.0 & 24.9 & 2.8 & 22.9 \\
\hline $3\left(10 \% 8^{\circ}\right)^{b}$ & 4.6 & 21.8 & 3.1 & 24.9 & 3.0 & 22.0 \\
\hline $4\left(10 \% 8^{\circ} \cup 5 \% 13^{\circ}\right)^{\mathrm{b}}$ & 4.9 & 22.2 & 3.4 & 25.3 & 3.2 & 22.3 \\
\hline $5\left(5 \% 13^{\circ}\right)^{\mathrm{b}}$ & 2.9 & 20.1 & 2.3 & 23.5 & 2.0 & 21.0 \\
\hline $6\left(10 \% 13^{\circ}\right)^{\mathrm{b}}$ & 2.3 & 17.8 & 1.6 & 22.4 & 1.6 & 20.1 \\
\hline $7((2 \cup 4) / 4)^{\mathrm{a}}$ & 5.6 & 22.8 & 4.0 & 25.9 & 3.9 & 23.4 \\
\hline $8(1 \cap 7)^{\mathrm{a}}$ & 4.2 & 22.0 & 3.5 & 25.6 & 3.1 & 22.6 \\
\hline
\end{tabular}

${ }^{a}$ Criterion that may, according to the author's interpretation of EN 15497, be used in production

${ }^{\mathrm{b}}$ Criterion that may not, on its own, be used in production according to EN 15497

within a finger joint. As shown by Table 4, criterion (4) on its own gave, for production case (a), slightly more waste than what criterion (2) did. The reason for this is, however, $n o t$ that criterion (4) in general gives larger margins to knots than what criterion (2) does (see Fig. 4), but that criterion (2), just as criterion (1), fails to ensure straight fibres in finger joints when knots are cut off just outside a board's end. Comparisons of results for criterion (3-6) give indications of how the percentage of waste changes due to changes of the definition of pronounced grain disturbance.

Production case (b) represents production of finger jointed structural timber of grade $\mathrm{C} 35$ where weak sections of boards are removed such that the remaining pieces can be utilized for production of finger jointed sawn timber of this high strength class. This means that the number of finger joints per unit length of produced timber is larger for production case (b) than what it is for production case (a). The average distance between finger joints was, for simulated production case (b) and finger joint criterion (1), $2.8 \mathrm{~m}$, whereas for production case (a) and finger joint criterion (1) the average distance was $4.2 \mathrm{~m}$. Hence the yield of produced timber is even more dependent on the finger joint criterion applied for production case (b) than what it is for production case (a). For example, for production case (b) the calculated waste was reduced by as much as $6.4 \%$ unit points when finger joint criterion (7), rather than criterion (1), was used.

Note that it is beyond the scope of this paper to investigate whether it is appropriate to produce strength graded sawn timber in the way as simulated by production case (b), i.e. to assign only parts of an investigated board to a certain strength class, but as explained above this actually occurs in production. Therefore, it is relevant to investigate the significance of the choice of finger joint criteria on such a production case. The results presented in Table 4 regarding the waste of production case (b) mean that the yield of timber in class C35 was, for the whole sample, 72-79\%, depending on the finger joint criteria used. This shall be compared to the yield obtained when discarding all boards that do not fulfil the requirement for class $\mathrm{C} 35$ along the entire length, in production of finger jointed $\mathrm{C} 35$ timber. For the investigated 
sample, this would be 50-53\% depending on the finger joint criteria used.

Timber of different origins and subsamples differ in quality and the percentages of waste differ between subsamples. For example, the subsample from mid Sweden gave, for production case (a) and using finger joint criteria (1) and (7), a waste of only $6.1 \%$ and $3.0 \%$, respectively, whereas the subsample from northern Sweden gave a waste of $9.8 \%$ and $5.4 \%$, respectively. On the other hand, for production case (b), the yield of the subsample from northern Sweden was higher than what the yield of the subsample from mid Sweden was. The explanation is that the timber from northern Sweden contained a high number of small knots leading to far-reaching restrictions on where finger joints may be placed (cf. criterion (1) in the example illustrated in Fig. 4c) and thus to considerable waste related to finger joints for connection of boards. At the same time, since the knots were small, comparatively few boards, or sections of boards of the subsample from northern Sweden needed to be discarded to meet the requirement defining the class $\mathrm{C} 35$. The number of boards of a single sample is relatively small, comprising between 90 boards (southern Sweden) and 294 boards (mid Sweden) and the results presented for the subsamples may not be representative for the regions they come from. Comparisons indicate, however, that the amount of waste related to finger jointing may differ considerably depending on the quality of the timber.

Table 5 shows how often application of the knot distance criteria, i.e. criteria (1-2), would imply violation of the criteria of pronounced grain disturbance, i.e. criteria (3-6). The percentage 13.8 given for production case (a) and criteria (1) and (3) in Table 5 means, for example, that $13.8 \%$ of the board ends, appropriate for finger jointing according to criterion (1), would mean violation with respect to criterion (3) if a finger joint was applied there. Results show that for production case (a), application of knot criterion (1) means

Table 5 Calculated percentage of how often a board end, cut to have sufficient margins to knot criterion 1 (margin of $3 \phi$ ) and knot criterion 2 (margin of $1.5 \phi$ ), respectively, contains skew fibres according to criterion 3-6 within the finger joint itself

\begin{tabular}{|c|c|c|c|c|}
\hline \multirow[t]{3}{*}{$\begin{array}{l}\text { Finger joint criterion } \\
\text { (fibre criterion) }\end{array}$} & \multicolumn{2}{|c|}{$\begin{array}{l}\text { Knot criterion } 1(3 \phi) \\
\text { Violation of fibre } \\
\text { criterion }[\%]\end{array}$} & \multicolumn{2}{|c|}{$\begin{array}{l}\text { Knot criterion } 2 \\
(1.5 \phi) \\
\text { Violation of fibre } \\
\text { criterion [\%] }\end{array}$} \\
\hline & \multicolumn{2}{|c|}{ Production case } & \multicolumn{2}{|c|}{ Production case } \\
\hline & (a) & (b) & (a) & (b) \\
\hline $3\left(10 \% 8^{\circ}\right)$ & 13.8 & 11.7 & 26.0 & 31.2 \\
\hline $4\left(10 \% 8^{\circ} \cup 5 \% 13^{\circ}\right)$ & 16.3 & 14.3 & 29.5 & 34.0 \\
\hline $5\left(5 \% 13^{\circ}\right)$ & 9.7 & 7.9 & 20.3 & 21.4 \\
\hline $6\left(10 \% 13^{\circ}\right)$ & 1.5 & 1.1 & 5.3 & 6.7 \\
\hline
\end{tabular}

Results presented for all 897 boards included in the investigation violation of fibre criteria (3-5) in as much as $10-16 \%$ of the cases. Only the very, so to speak, generous fibre criterion (6) is seldomly violated applying criterion (1). For production case (b) the application of knot criterion (1) does not mean violation of fibre criteria (3-5) quite as often as it does for production case (a) but still quite often, namely in $8-14 \%$ of the cases. Application of knot criterion (2) would mean violation of the fibre criteria more than twice as often as what application of knot criterion (1) does. However, criterion (2) is not allowed to be used on its own, but only in combination with a fibre criterion.

\section{Conclusion}

Finger joints in structural timber shall be placed in clear wood where fibres are parallel to the longitudinal direction of the connected members. In the European standard EN 15497 , requirements are expressed in terms of a minimum distance between the finger joint and knots in the assembled members. When only using knot dimensions, a margin of three times the knot diameter is required. This margin may, however, be reduced to 1.5 times the knot diameter if an appropriate automated system guarantees that in the range of the finger joint the grain orientation is parallel to the longitudinal direction of the board, as it is formulated in the standard. Thus, appropriate application of finger joints at a minimum of waste requires accurate detection of both knots and fibre orientation. The aim of the present research was to

- develop a procedure for accurate identification of knots on Norway spruce timber on the basis of surface scanning,

- make reasonable interpretations of the requirements of EN 15497 regarding unacceptable fibre deviations within finger joints of structural timber,

- calculate the yield of different production cases of finger jointed structural timber, applying alternative criteria for where finger joints are allowed with respect to distance to knots and fibre distortions.

It was concluded that

- accurate and robust detection of knots on wood surfaces can be done on the basis of knowledge of fibre orientation on four wood surfaces obtained by laser scanning in combination with photographs, i.e. high resolution data of light intensity of the same four surfaces, all of which can be obtained in high speed using an industry timber scanner,

- when interpreting the requirement of EN 15497, i.e. that no pronounced grain disturbance is allowed within finger joints, the following was taken into account: first, at 
what fibre angle tensile strength is substantially reduced (compared to the strength of straight fibres) and second, that inclined fibres may occur in the timber independent of knots. Regarding tensile strength, a fibre angle of $8^{\circ}$ means, according to earlier research, that the tensile strength is reduced by about $50 \%$. Fibre angles smaller than $6^{\circ}-8^{\circ}$, on the other hand, are sometimes caused by one or several of the following causes; spiral grain, skew sawing, taper and lack of accuracy of the detection of fibre orientation. Therefore, the smallest fibre angle considered herein in criteria for pronounced grain disturbance was $8^{\circ}$.

- when using finger joints simply to produce timber of long lengths, the percentage of wasted material decreases, according to simulations, from about $7.4 \%$ to about $4.0 \%$, when using a combined criterion on fibre disturbance and a requirement of a margin of 1.5 times the knot diameter, rather than the simpler criterion of three times the knot diameter. If finger joints are also used to re-connect parts of boards after removal of weak sections within them, for example to produce timber of a certain strength class, even larger savings can be made.

Further research should be carried out to investigate how the strength of finger joints is affected by small fibre distortions within the joints. If, for example, strength of finger joints is significantly affected by fibre angles smaller than, for example, $8^{\circ}$ over a certain part of the finger-joint, stricter requirements on fibre orientation should be considered. As a result, knowledge of fibre orientation would then lead to increased characteristic strength of finger joints rather than to increased yield in production. It would then also be possible to optimize between yield and characteristic strength depending on the requirements of the timber produced.

Acknowledgments Open access funding provided by Linnaeus University.

\section{Compliance with ethical standards}

Conflict of interest On behalf of all authors, the corresponding author states that there is no conflict of interest.

Open Access This article is distributed under the terms of the Creative Commons Attribution 4.0 International License (http://creativeco mmons.org/licenses/by/4.0/), which permits unrestricted use, distribution, and reproduction in any medium, provided you give appropriate credit to the original author(s) and the source, provide a link to the Creative Commons license, and indicate if changes were made.

\section{References}

Aicher S (2003) Structural adhesive joints including glued-in bolts. In: Thelandersson S, Larsen HJ (eds) Timber engineering. Wiley, Chichester, pp 333-363

Bacher M (2008) Comparison of different machine strength grading principles. COST E53, 29-30 October 2008, Delft, The Netherlands, pp 183-193

Briggert A, Olsson A, Oscarsson J (2016) Three-dimensional modelling of knots and pith location in Norway spruce boards using tracheid-effect scanning. Eur J Wood Prod 74(5):725-739

Dinwoodie JM (2000) Timber: Its nature and behaviour. E \& FN Spon, New Fetter Lane, London

EN 14081-2 (2010+A1:2012) Timber structures-strength graded structural timber with rectangular cross section-Part 2: Machine grading; additional requirements for initial type testing. European Committee for Standardization, Brussels, Belgium

EN 384 (2010) Structural timber-determination of characteristic values of mechanical properties and density. European Committee for Standardization

EN 408 (2010+A1:2012) Timber structures-structural timber and glued laminated timber-determination of some physical and mechanical properties. European Committee for Standardization

EN 14080 (2013) Timber structures_glued laminated timber and glued solid timber-requirements. European Committee for Standardization, Brussels

EN 14081-1 (2016) Timber structures-strength graded structural timber with rectangular cross section-Part 1: General requirements. European Committee for Standardization, Brussels

EN 14081-2 (2018) Timber structures-strength graded structural timber with rectangular cross section-Part 2: Machine grading; additional requirements for initial type testing. European Committee for Standardization, Brussels

EN 15497 (2014) Structural finger jointed solid timber-performance requirements and minimum production requirements. European Committee for Standardization, Brussels

EN 338 (2016) Structural timber-strength classes. European Committee for Standardization, Brussels

Fink G, Frangi A, Kohler J (2015) Bending tests on GLT beams having well-known local material properties. Mater Struct 48:3571-3584

Frese M, Blass HJ (2009) Bending strength of spruce glulam. Eur J Wood Prod 67:277-286

Gustafsson PJ (2003) Fracture perpendicular to grain—structural applications. In: Thelandersson S, Larsen HJ (eds) Timber engineering. Wiley, Chichester, pp 103-130

Hanhijärvi A, Ranta-Maunus A (2008) Development of strength grading of timber using combined measurement techniques. Report of the Combigrade-project-phase 2. VTT Publication 686

Hankinson RL (1921) Investigation of crushing strength of spruce at varying angles of grain. Air Service Information Circular, 3(259), Material Section Report No 130, US Air Service, USA

Kandler G, Lukacevic M, Füssl J (2016) An algorithm for the geometric reconstruction of knots within timber boards based on fibre angle measurements. Constr Build Mater 124:945-960

Matthews PC, Beech BH (1976) Method and apparatus for detecting timber defects. US Patent no. 3676384A

Olsson A, Oscarsson J (2017) Strength grading on the basis of high resolution laser scanning and dynamic excitation: a full scale investigation of performance. Eur J Wood Prod 75(1):17-31

Olsson A, Oscarsson J, Serrano E, Källsner B, Johansson M, Enquist B (2013) Prediction of timber bending strength and in-member cross-sectional stiffness variation on the basis of local wood fibre orientation. Eur J Wood Prod 71(3):319-333 
Ormarsson (1999) Numerical analysis of moisture-related distortions in sawn timber. Doctoral thesis, 99:7 Department of Structural Mechanics, Chalmers University of Technology, Gothenburg

Säll H (2002) Spiral grain in Norway spruce. Doctoral thesis, Acta Wexionesia No 22/2002, Växjö University Press, Växjö University, Växjö, Sweden

Seltman J (1992) Indication of slope-of-grain and biodegradation in wood with electromagnetic waves. In: Lindgren O (ed) First international seminar on scanning technology and image processing on wood. Luleå University of Technology, Skellefteå

Serrano E. (2000) Adhesive joints in timber engineering - modelling and testing of fracture properties. Doctoral thesis, Report TVSM1012, Lund University, Lund, Sweden

Soest J, Matthews PC, Wilson B (1993) A simple optical scanner for grain defects. In: Proceedings of 5th International Conference on Scanning Technology and Process Control for the Wood Products Industry, October 25-27, Atlanta, USA
SS 230120 (2010) Nordic visual strength grading rules for timber (INSTA 142). Swedish Standard Institute, Stockholm

Thunell B (1981) Instruktion för sortering och märkning av T-virke, femte upplagan (Instructions for grading and marking of T classed sawn timber, 5th edn. T-virkesföreningen, Stockholm

WoodEye AB (2018) WoodEye. http://woodeye.se/en/. Accessed 27 Aug 2018

Ziethén R (2017) personal communication with Rune Ziethén of RISE-the Swedish Research Institute, June 12, 2017

Publisher's Note Springer Nature remains neutral with regard to jurisdictional claims in published maps and institutional affiliations. 